\title{
The Wavelet Analysis of the Vibroacoustic Signal Measurement in a Transient State Operation of Power Transformer
}

\author{
S. Borucki*, A. Cichon, T. Boczar And D. ZMARZly \\ Faculty of Electrical Engineering, Automatic Control and Computer Science, Opole University of Technology \\ Proszkowska 76, 45-758 Opole, Poland
}

\begin{abstract}
The article presents the results of attempts made to develop a non-invasive diagnostic method of a technical state of a transformer's core based on an analysis of mechanical vibrations registered during the start-up of the transformer analyzed. The paper presents a characteristic of the transformer under study, the measuring systems applied and the method developed for assessing the degree of the core loosening. The original results presented in the paper have been obtained under laboratory conditions during tests conducted on an oil transformer of $200 \mathrm{kVA}$. The analysis of recorded virboacoustic signals was performed by means of discrete wavelet transform for three different operating cases: the transformer with no core defect, the transformer with loose screws of the magnetic circuit of the upper yoke, and the transformer with completely loosen screws of the beam of the upper yoke and the lower core. The signals have been recorded on two perpendicular measuring axes: $0 X$ and $0 Y$ and their measured values were subject to the wavelet analysis.
\end{abstract}

PACS: 43.40.-r, 43.40.Vn, 43.58.Wc

\section{Introduction}

The basic element of a transformer's construction is the core. A high reliability of its functioning determines a long-term and a problem-free operation of high power block and network units. When the magnetic circuit sheet pack of high power transformers loosens, eddy currents of high value occur and in consequence local overheatings may be observed as well as a rapid increase in an oil temperature inside a transformer's tank. An operation of the transformer with a growing defect in the structure of the core which lasts many months not only considerably accelerates ageing processes of an isolation system but it can also causes a sudden increase in the concentration of flammable gases and an emergency shutdown of the device by a gas-circuit breaker. The diagnostics of the magnetic circuit of the units operating in the power system is currently based, to a great extent, on the dissolved gas analysis (DGA) test results of oil [1, 2], measurements of magnetizing currents $[3,4]$, vibroacoustic measurements under steady operating conditions of the transformer $[5,6]$ and measurements of the corrected sound pressure level [7-11].

The subject area of this paper concerns an assessment of the technical condition of the transformers' cores based on the measurement and the analysis of vibroacoustic

* corresponding author; e-mail: s.borucki@po.opole.pl signals registered when switching the transformers to an idle work mode. The issue dealt with herein is a further continuation of the previous research work on an application of so-called "modified vibroacoustic method under industrial conditions" which had been carried out by the team of the Institute of Electric Power Engineering of Opole University of Technology for several years. Their results are presented in the following publications [12-14]. The main aim of conducted research, whose results are presented herein, is to offer a new diagnostic method enabling to evaluate the transformer's magnetic circuit technical condition (to identify its loosening) on the basis of discrete wavelet transform (DWT) analysis results of the vibrations registered during the start-up of the device under study.

\section{Methodology of the experiment}

The laboratory trials were run on a unit with an oil insulation in order to identify the application possibility of DWT transform results of vibroacoustic signals registered with the modified vibroacoustic method for the cores diagnostics. The transformer under study is characterised by the following: type - TZ-200/20, power $200 \mathrm{kVA}$, primary voltage - $15750 \mathrm{~V}$, secondary voltage - $400 \mathrm{~V}$, scheme of connections - Yz5, cooling AN, total weight $-1430 \mathrm{~kg}$, year of production - 1959 . The experiment comprised vibroacoustic measurements conducted on the transformer in its three different operating cases. The first case related to the measurements 
of vibrations during the start-up phase of the unit with a properly twisted core. The second case was connected with the vibrations registration while switching on the unit with loose screws of the upper yoke (Ist degree defect - Fig. 1a), whereas the third stage of the measurements was carried out at the start-up stage with simultaneous loosening of the screws pressing the beams of the upper and lower yoke (IInd degree defect - Fig. 1b).

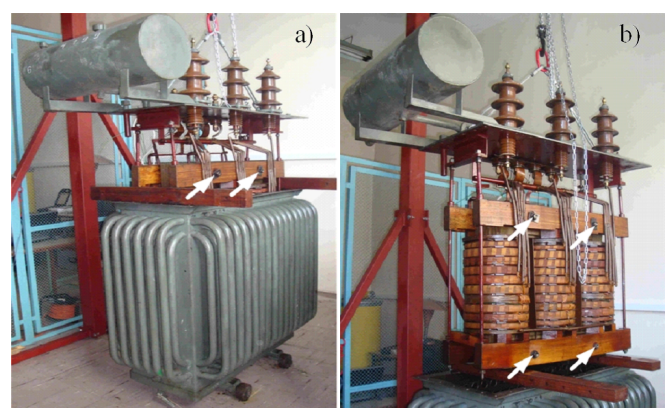

Fig. 1. Tested transformer with indicated points of the introduced core's defects: (a) Ist degree defect, (b) IInd degree defect.

Vibroacoustic signals of the oil transformer have been registered with two uniaxial accelerators of 4515 type from Brüel \& Kjær, which were placed on the axis of symmetry on the transformer's side walls. The first accelerator (Acc1) was placed in the centre point of the side wall with the medium voltage feedthrough outlets, whereas the other accelerator (Acc2) was placed on the side wall surface on the L1 phase column side (Fig. 2).

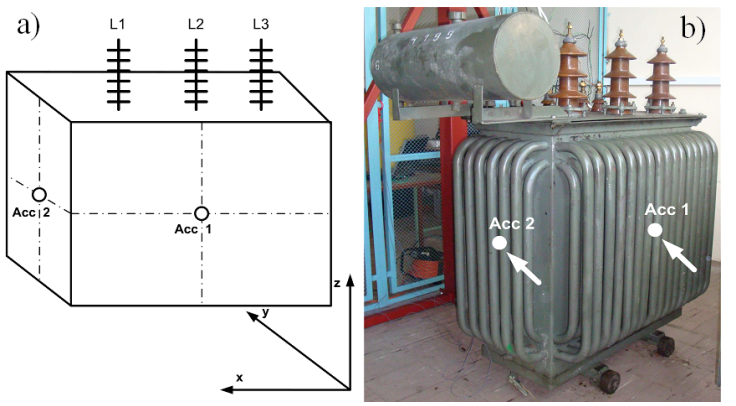

Fig. 2. Location of the accelerators in the oil transformer's tank: (a) pictorial drawing, (b) real location of registering points.

The signal received by the accelerometers was transmitted onto the inputs of the measuring and registering apparatus which consisted of the system Pulse DYN-XI from Brüel \& Kjær. The total registration time of mechanical vibrations from the moment the transformer was switched onto the point it worked in a steady mode was determined as $t=10 \mathrm{~s}$ and the value of sampling frequency was set to be $f=32768 \mathrm{~Hz}$. The measured signals were subject to time-frequency analysis with the use of DWT transform. In order to complete a multi- -resolution wavelet analysis DWT, Matlab environment was applied.

\section{The analysis of the results obtained}

DWT analysis of mechanical vibrations generated when the analysed transformer was switched on in a transcent work mode was completed at seven levels of a decomposition. The table below presents the scopes corresponding to bandwiths of the filters of analysed details.

Frequency bands of DWT analysis details.

TABLE

\begin{tabular}{c|c|c}
\hline \hline Detail & $\begin{array}{c}\text { Medium frequency } \\
{[\mathrm{kHz}]}\end{array}$ & $\begin{array}{c}\text { Frequency bandwidth } \\
{[\mathrm{kHz}]}\end{array}$ \\
\hline D1 & 12288 & $8192-16384$ \\
D2 & 6144 & $4096-8192$ \\
D3 & 3072 & $2048-4096$ \\
D4 & 1536 & $1024-2048$ \\
D5 & 768 & $512-1024$ \\
D6 & 384 & $256-512$ \\
D7 & 192 & $128-256$
\end{tabular}

In the analysis, the wavelet as orthogonal basis functions of Symmlet 8 have been applied. The functions are characterised by a precise symmetry and a linear phase. Detailed characteristics of the wavelet base including its time course, scaling function, tree-based decomposition scheme and a graph illustrating the frequency-scale relations are presented among others in paper [15].

Exemplary results of registered vibrations subject to DWT analysis are presented in Figs. 3-5. Graphs marked as (a) concern the measurements taken with the accelerometer Acc1 and marked as (b) with the accelerometer Acc2. Figure 3 presents the results of a multi-resolution DWT analysis of signals registered during the start-up of the device of $200 \mathrm{kVA}$ with no core defect. The wavelet decomposition of mechanical vibrations registered on two perpendicular measuring axes showed an activity within the frequencies corresponding with all the details. The highest amplitude was noted for the waveforms determined at levels D3-D7, that is the components of the bandwidth 128-4096 Hz. Once the device is switched on, high-frequency acoustic signals are generated. Their occurrence may be observed as impulse changes of the values of details D1-D2. However, the shapes of the wavelet structures within the following band 4096-16384 are characterised by a considerably lower energy level.

The DWT analysis results shown in Fig. 3a and b proved that the shapes at different levels of decomposition D1-D4 are similar in terms of their amplitudes and times of their occurrence regardless of the measuring axis. The differences in a time-frequency image of the vibrations recorded with the accelerometers Acc1 and Acc2 are observed mainly in the shapes of details D6-D7. However, it does not affect further comparative analysis of the results interpretation and an assessment of the transformer's core technical condition when its mechanical construction is considered. In the process of identifying the magnetic circuit's defects the important role is 
played by fluctuations and changes in the values of details D1-D5 especially from the moment the voltage supply is switched on up to $400 \mathrm{~ms}$.

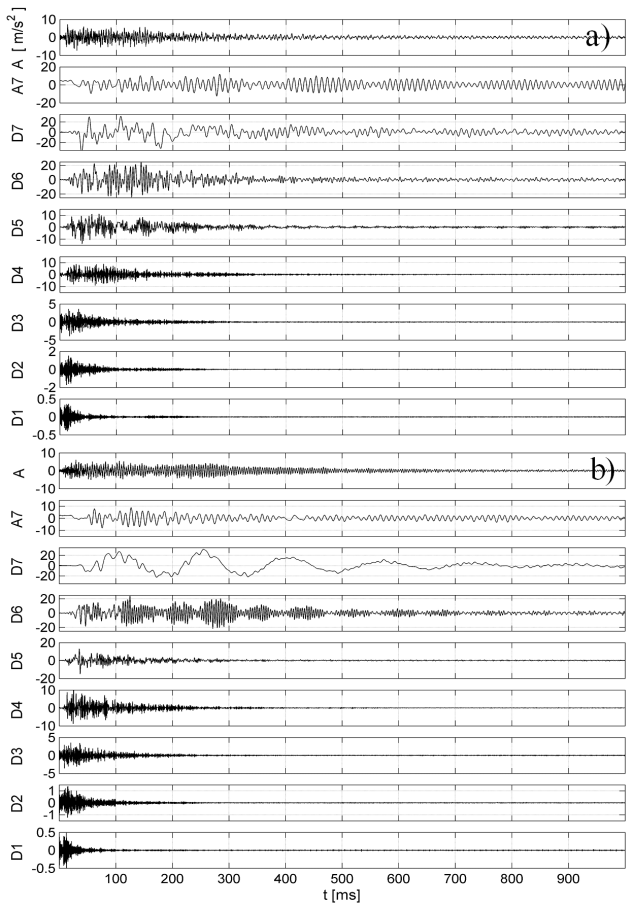

Fig. 3. Results of the wavelet decomposition of the transformer vibrations measured for the oil transformer with no core defect: (a) measurement with accelerator Acc1, (b) measurement with accelerator Acc2.

Figure 4 presents the shape of a wavelet decomposition of the vibrations registered during tests conducted on a device with a modelled defect of the $\mathrm{I}^{\circ}$. DWT analysis of measured signals in such case showed noticeable decrease in amplitude for details D1-D2 in a determined time range (Fig. 4a,b). The decreased power share of the wavelet structures within the ranges 4096-8192 and $8192-16384 \mathrm{~Hz}$ proves that the frequency bandwidth of registered vibrations is limited. The comparison of the results obtained with the results registered in case of the transformer with no defect (Fig. 3) revealed a significant increase in detail's D7 value. Amplitude fluctuations are also slightly different at the 6th level of decomposition. The analysis of the measurements conducted with the accelerator Acc1 (Fig. 4a) showed a slight increase in the acitivity of detail D6. When the accelerator Acc2 was applied, a significant decrease in an amplitude modulation of this structure was observed. Having compared the DWT results of the vibroacoustic signals recorded on the transformer with no defect (Fig. 3) and the transformer with $\mathrm{I}^{\circ}$ defect (Fig. 4), it may be stated that the fluctuations of details D3-D5 are similar in their values and shapes in time.

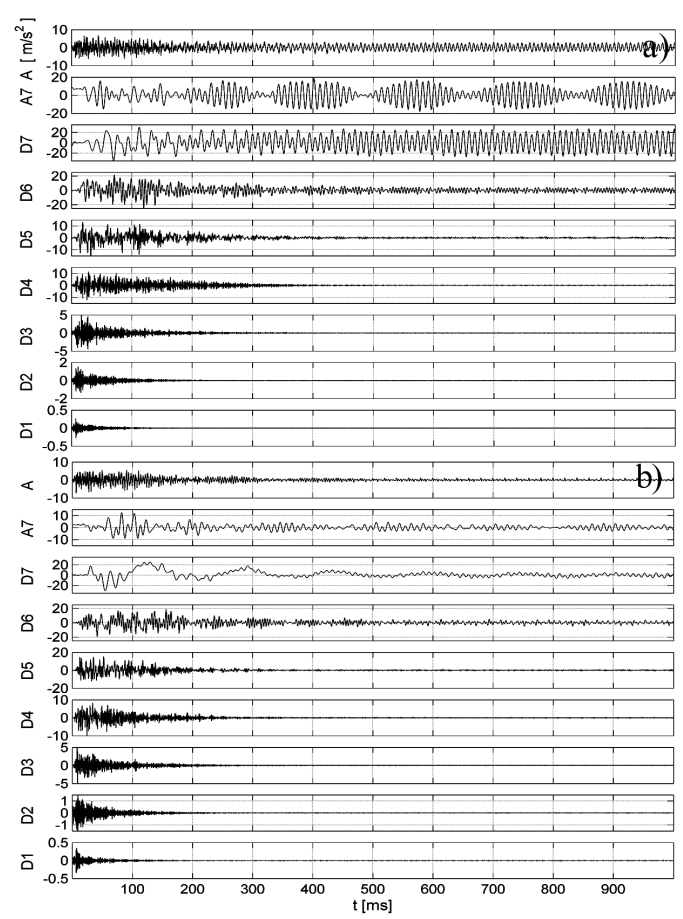

Fig. 4. Results of the wavelet decomposition of the transformer vibrations measured for the oil transformer with $I^{\circ}$ core defect: (a) measurement with accelerator Acc1, (b) measurement with accelerator Acc2.

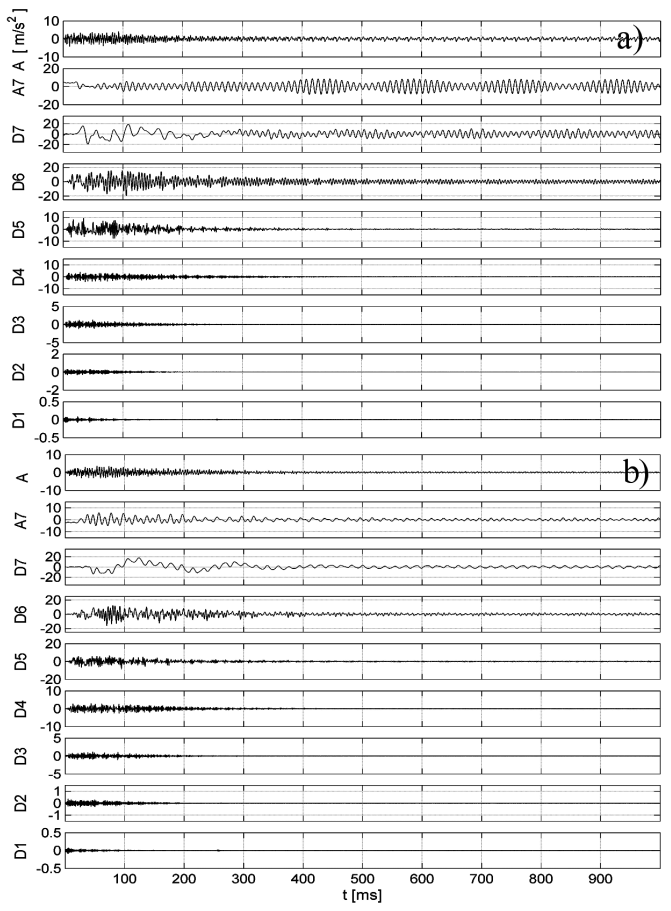

Fig. 5. Results of the wavelet decomposition of the oil transformer with $\mathrm{II}^{\circ}$ core defect: (a) measurement with accelerator Acc1, (b) measurement with accelerator Acc2. 
Figure 5 presents time course, an approximation at the 7th level of decomposition and details D1-D7, determined on the basis of the signals analysis, which were recorded on the transformer with loosen upper and lower part of the magnetic circuit. DWT vibration analysis of the unit with $\mathrm{II}^{\circ}$ defect showed further decrease in the values of details D1-D2 and a significant decrease in the amplitude of structures at third, fourth and fifth level. Decreased power share of the shapes D1-D5 proves the frequency bandwidth of recorded vibrations to be significantly narrowed. DWT wavelet decomposition of the vibrations registered on the unit with $\mathrm{II}^{\circ}$ defect has also proved that the limitation of the frequency bandwidth dominant in a spectrum of analysed signals has an effect which may be observed at the same time in characteristics obtained when data measured on $0 Y$ and $0 X$ axes are processed digitally (Fig. 5a and b).

\section{Summing-up}

DWT analysis of vibroacoustic signals registered at the start-up of the oil transformer revealed the presence of different structures at different levels of the wavelet decomposition. Having compared the results of DWT transform, it has been observed that with the growing degree of the magnetic circuit defect, a gradual reduction of the power share in the frequency band corresponding with filtration at the level of details D1-D2 (Fig. 4) took place followed with D1-D5 (Fig. 5). Similar tendency has been observed in case of vibrations registered on two perpendicular measuring axes. DWT analysis of the signals registered with the modified vibroacoustic method enables to identify defects of the power transformer core.

The authors of the paper, in reference to the results presented herewith, have demonstrated a potential possibility of applying the results of the time-frequency analysis DWT to assess technical condition of the power transformers cores by means of the modified vibroacoustic method. The results obtained during the experiment conducted within the research show then the possibility of developing and implementing a non-destructive and effective method of evaluating the technical condition of magnetic circuit mechanical construction of the transformers in service, in particular of those with a strategic meaning.

\section{Acknowledgments}

The work was financed with the means from the National Research and Development Center within LIDER program.

\section{References}

[1] M. Duval, IEEE Electr. Insulat. Mag. 18, 8 (2002).

[2] S.S. Rajurkar, A.R. Kulkami, J.G. Nandapurkar, in: Proc. 16th National Power Sys. Conf., India, Osmania University, Hyderabad (India) 2010, p. 180.

[3] W. Matulewicz, Diagnostics of Power Transformer, Publish. of Gdańsk UT, Gdańsk 1998 (in Polish).

[4] Framework Manual Operation of Power Transformers, Energopomiar-Elektryka, Gliwice 2006 (in Polish).

[5] S. Borucki, A. Cichoń, Electr. Rev. 86 (7), 45 (2010) (in Polish).

[6] T. Boczar, S. Borucki, A. Cichoń, M. Lorenc, Acta Phys. Pol. A 114, A21 (2008).

[7] D. Snell, J. Magn. Magn. Mater. 320, 535 (2008).

[8] J. Ignac-Nowicka, T. Pustelny, Z. Opilski, W. Maciak, W. Jakubik, M. Urbanczyk, Opt. Eng. 42, 2978 (2003).

[9] T. Pustelny, J. Ignac-Nowicka, Z. Opilski, Opt. Appl. 34, 563 (2004).

[10] S. Borucki, T. Boczar, A. Cichon, Arch. Acoust. 36, 49 (2011).

[11] S. Borucki, T. Boczar, A. Cichoń, Arch. Acoust. 32, Supplement, 291 (2007).

[12] S. Borucki, Acta Phys. Pol. A 116, 277 (2009).

[13] S. Borucki, Acta Phys. Pol. A 120, 571 (2011).

[14] S. Borucki, A. Cichoń, J. Subocz, E. Kornatowski, Electr. Rev. 86 (11b), 22 (2010) (in Polish).

[15] L. Debnath, Wavelet Transform and Time-Frequency Analysis, Birkhauser, Boston 2001. 